\title{
ASYMPTOTIC PROPERTIES OF PROTECTED NODES IN RANDOM RECURSIVE TREES
}

\author{
HOSAM M. MAHMOUD, ${ }^{*}$ The George Washington University \\ MARK D. WARD, ${ }^{* *}$ Purdue University
}

\begin{abstract}
We investigate protected nodes in random recursive trees. The exact mean of the number of such nodes is obtained by recurrence, and a linear asymptotic equivalent follows. A nonlinear recurrence for the variance shows that the variance grows linearly, too. It follows that the number of protected nodes in a random recursive tree, upon proper scaling, converges in probability to a constant.
\end{abstract}

Keywords: Recursive tree; random structure; combinatorial probability

2010 Mathematics Subject Classification: Primary 60C05; 60F05

\section{Introduction}

In several very recent papers protected nodes in certain classes of random trees were studied. Cheon and Shapiro [2] investigated the average number of protected nodes in unlabeled ordered trees and in Motzkin trees. Mansour [6] considered the average number of protected nodes in $k$-ary trees, Du and Prodinger [3] analyzed the average number of protected nodes in random digital trees, and Mahmoud and Ward [5] presented a central limit theorem, as well as exact moments of all orders, for the number of protected nodes in binary search trees.

In this paper we investigate the average number of protected nodes in yet another class of random tree: the random recursive tree. The random recursive tree is a naturally growing structure that underlies several stochastic developments, such as recruiting, the spreading of chain letters, contagion, and the evolution of the union-find algorithm. Smythe and Mahmoud [7] provides a survey of results and numerous applications of recursive trees.

A recursive tree of $n$ nodes grows randomly as follows. The labels $1,2, \ldots, n$ arrive sequentially. Initially the tree is empty. When label 1 arrives, a tree of one root node (labeled with 1 ) is created. Subsequently, the label 2 arrives and a child node (labeled with 2 ) is adjoined to the root. Upon arrival of label 3, a node labeled with 3 joins the tree by choosing as its parent one of the two labeled nodes in it with equal probability. The process goes forth in a like manner: For $i=2,3, \ldots, n$, after $i-1$ insertion steps, there is a random recursive tree of size $i-1$. When the $i$ th node appears, a node labeled with $i$ joins the tree by randomly choosing as its parent any of the existing nodes (with equal probability).

The growth algorithm of random recursive trees induces a uniform distribution on the trees: all $(n-1)$ ! recursive trees of size $n$ are equally likely. Many important properties of recursive trees have been analyzed from the the viewpoint of recurrence occurring naturally

Received 1 May 2013; revision received 23 February 2014.

* Postal address: Department of Statistics, The George Washington University, Washington, DC 20052, USA.

Email address: hosam@gwu.edu

** Postal address: Department of Statistics, Purdue University, West Lafayette, IN 47907-2067, USA. Email address: mdw@purdue.edu 


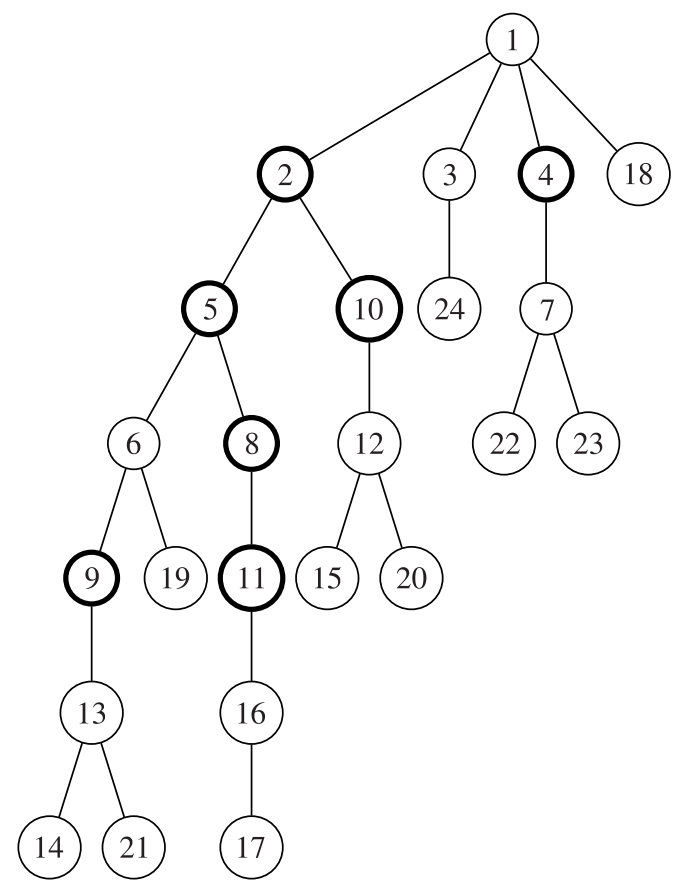

FIGURE 1: Example of a recursive tree of size 24 with protected nodes shown in bold.

in the stochastic growth. Alternatively, the uniform distribution of the trees is amenable to analytic generating functionology [1].

A node with no descendants in a tree is a leaf. In a rooted tree of whatever kind, a node is protected if its distance (number of edges) from all the leaves in the subtree it roots is at least two. Figure 1 shows a recursive tree of size 24; protected nodes are represented as bold circles.

\section{The average number of protected nodes in recursive trees}

Let $X_{n}$ be the number of protected nodes in a random recursive tree of size $n$. In the tree of Figure $1, X_{24}$ is 7 . We will use a decomposition of a recursive tree introduced in [8]. Remove the special edge joining the nodes labeled 1 and 2 . The tree then falls apart into a forest of two trees, one is rooted at 2 , which we will recognize as a special subtree of the original recursive tree; the other tree of the forest is a tree rooted at 1 , which is a nonspecial subtree. Let $U_{n}$ be the size of the special subtree, and so $n-U_{n}$ is the size of the nonspecial subtree. It is shown in [8] that $U_{n}$ has a uniform distribution on $\{1,2, \ldots, n-1\}$. Note that the special (nonspecial) subtree is isomorphic to a recursive tree of size $U_{n}$ (size $n-U_{n}$ ) that has the same uniform probability of a random recursive tree of that size. Also, the two subtrees are conditionally independent (given $U_{n}$ ).

We will make use of two events. Let $\mathcal{R}_{n}$ be the event that the root node of a recursive tree is protected, and let $\mathcal{Q}_{n}$ be the event that the nonspecial subtree, as it stands alone as a tree in the forest, has a protected root. We will also express the formulation in terms of the indicator notation: for any event $\mathcal{E}$, the indicator $\mathbf{1}_{\mathcal{E}}=1$, if $\mathcal{E}$ occurs, and $\mathbf{1}_{\mathcal{E}}=0$, otherwise. 
Random recursive trees of size $n \leq 2$ do not have any protected nodes. So, $X_{0}=X_{1}=$ $X_{2} \equiv 0$. For $n \geq 3$, we have a stochastic recurrence for $X_{n}$ : it is the number of protected nodes in the special and nonspecial subtrees, and we need a few adjustments according to the condition of the root of the tree before the special edge was removed. When we attach the two trees of the forest together to reconstruct the original recursive tree (i.e. when we put back the special edge), we add 1 if $U_{n}=n-1 \geq 2$, a case where the nonspecial subtree consists of a single unprotected node (labeled with 1); when we join the trees of the forest by adding back the special edge, the root of the original recursive tree becomes protected. On the other hand, if the root of the nonspecial subtree is protected, this protection is lost after attaching the special edge in the extremal case when $U_{n}=1$.

For $n \geq 3$, we have a stochastic recurrence, which gives rise to an equality:

$$
X_{n}=X_{U_{n}}+\tilde{X}_{n-U_{n}}+\mathbf{1}_{\left\{U_{n}=n-1\right\}}-\mathbf{1}_{\left\{U_{n}=1\right\} \cap Q_{n}} ;
$$

the tilded random variable $\widetilde{X}_{n-U_{n}}$ is conditionally independent of $X_{U_{n}}$ (given $U_{n}$ ). Note also that, for each $j \geq 0, \widetilde{X}_{j}$ has the same distribution as $X_{j}$.

Theorem 2.1. Let $X_{n}$ denote the number of protected nodes in a random recursive tree. Then we have

$$
\mathbb{E}\left[X_{n}\right]=\left(\frac{1}{2}-\frac{1}{\mathrm{e}}\right) n+n \sum_{k=n+1}^{\infty} \frac{(-1)^{k}}{k !} \text { for } n \geq 3 .
$$

Proof. Taking the expectation of the stochastic recurrence (2.1), we obtain a recurrence for the mean. By the symmetry of the distribution of the sizes of the special and nonspecial subtrees, we can condition the right-hand side on $U_{n}=k$ and obtain

$$
\mathbb{E}\left[X_{n}\right]=\frac{2}{n-1} \sum_{k=1}^{n-1} \mathbb{E}\left[X_{k}\right]+\frac{1}{n-1}-\mathbb{P}\left(\left\{U_{n}=1\right\} \cap \mathcal{Q}_{n}\right),
$$

valid for $n \geq 3$. Let $\mathcal{R}_{j}$ be the event that a random recursive tree built on $j$ nodes has a protected root node. The joint event $\left\{U_{n}=1\right\} \cap \mathcal{Q}_{n}$ occurs when the special tree is a single node (labeled with 2) and the nonspecial subtree (independently) forms a tree with a protected root node. That is to say, the following relation holds:

$$
\mathbb{P}\left(\left\{U_{n}=1\right\} \cap \mathcal{Q}_{n}\right)=\frac{1}{n-1} \mathbb{P}\left(\mathcal{R}_{n-1}\right) .
$$

It is evident that we need to obtain the probability that the root node is protected in a recursive tree. Observe that

$$
\mathcal{R}_{n}=\left(\left\{U_{n} \geq 2\right\} \cap \mathcal{R}_{n-U_{n}}\right) \cup\left\{U_{n}=n-1\right\},
$$

valid for $n \geq 2$, and that the two events $\left\{U_{n} \geq 2\right\}$ and $\mathcal{R}_{n-U_{n}}$ are conditionally independent. And so, we obtain the required probability by writing a recurrence and solving it. Condition on $U_{n}=k$ to write

$$
\mathbb{P}\left(\mathcal{R}_{n}\right)=\frac{1}{n-1} \sum_{k=2}^{n-1} \mathbb{P}\left(\mathcal{R}_{n-k}\right)+\frac{1}{n-1} .
$$

We solve the latter recurrence by differencing. Write a version of the recurrence with $n-1$ replacing $n$ (and, thus, is valid only for $n \geq 3$ ), and subtract it from the original version. The telescoping sums cancel out, leaving only the single term

$$
(n-1) \mathbb{P}\left(\mathcal{R}_{n}\right)-(n-2) \mathbb{P}\left(\mathcal{R}_{n-1}\right)=\mathbb{P}\left(\mathcal{R}_{n-2}\right),
$$


valid for $n \geq 3$. This recurrence has the (MAPLE $^{\circledR}$-assisted and checkable-by-induction) solution

$$
\mathbb{P}\left(\mathcal{R}_{n}\right)=\frac{\Gamma(n,-1)}{\mathrm{e} \Gamma(n)} \quad \text { for } n \geq 2,
$$

where $\Gamma(\cdot)$ is the usual Gamma function and

$$
\Gamma(y, s)=\Gamma(y)+\int_{v=s}^{0} v^{n-1} \mathrm{e}^{-v} \mathrm{~d} v .
$$

After substituting (2.4) in (2.3), then substituting the new form of (2.3) in (2.2), the latter can be solved. For $n \geq 4$, by differencing we obtain

$$
(n-1) \mathbb{E}\left[X_{n}\right]=n \mathbb{E}\left[X_{n-1}\right]-a_{n},
$$

where

$$
a_{n}=\mathbb{P}\left(\mathcal{R}_{n-1}\right)-\mathbb{P}\left(\mathcal{R}_{n-2}\right)=\frac{(-1)^{n-2}}{(n-2) !} .
$$

Subsequently, the solution to recurrence (2.2), for $n \geq 4$, is

$$
\mathbb{E}\left[X_{n}\right]=\frac{n}{6}-n \sum_{k=3}^{n-1} \frac{(-1)^{k+1}}{(k+1) !}=\left(\frac{1}{2}-\frac{1}{\mathrm{e}}\right) n+n \sum_{k=n+1}^{\infty} \frac{(-1)^{k}}{k !} .
$$

The equation also holds for the $n=3$ case.

Corollary 2.1. Asymptotically, we have

$$
\mathbb{E}\left[X_{n}\right]=\left(\frac{1}{2}-\frac{1}{\mathrm{e}}\right) n+O\left(\frac{1}{n !}\right) \text { as } n \rightarrow \infty .
$$

According to Corollary 2.1 , on average only about $13 \%$ of the nodes of a large random recursive tree are protected.

\section{The variance}

In this section we compute linear bounds on the variance. Like in the case of the mean, these bounds are asymptotically computed by recurrence. We briefly outline the lengthy steps in the second moment calculation. We start with squaring both sides of (2.1). On the righthand side we obtain the squares of each individual term (and, of course, the square of each indicator is itself) and the cross-product of terms. For $n \geq 3$, three of the cross-product terms are identically 0 . These are

- the term $2 \mathbf{1}_{\left\{U_{n}=n-1\right\}} \mathbf{1}_{\left\{U_{n}=1\right\} \cap Q_{n}}=0$, as $U_{n}$ cannot be, for $n \geq 3$, equal to 1 and to $n-1$ at the same time;

- the term $2 X_{U_{n}} \mathbf{1}_{\left\{U_{n}=1\right\} \cap Q_{n}}=0$, for the $U_{n}=1$ case, we must have $X_{U_{n}}=X_{1}=0$;

- the term $2 \tilde{X}_{n-U_{n}} \mathbf{1}_{\left\{U_{n}=n-1\right\}}=0$, because when $U_{n}=n-1, \widetilde{X}_{n-U_{n}}=\widetilde{X}_{1}=0$.

Taking expectations of what is left, we obtain the recurrence

$$
\begin{aligned}
\mathbb{E}\left[X_{n}^{2}\right]= & \mathbb{E}\left[X_{U_{n}}^{2}\right]+\mathbb{E}\left[X_{n-U_{n}}^{2}\right]+\mathbb{E}\left[\mathbf{1}_{\left\{U_{n}=n-1\right\}}\right]+\mathbb{E}\left[\mathbf{1}_{\left\{U_{n}=1\right\} \cap Q_{n}}\right]+2 \mathbb{E}\left[X_{U_{n}} \tilde{X}_{n-U_{n}}\right] \\
& +2 \mathbb{E}\left[X_{U_{n}} \mathbf{1}_{\left\{U_{n}=n-1\right\}}\right]-2 \mathbb{E}\left[\tilde{X}_{n-U_{n}} \mathbf{1}_{\left\{U_{n}=1\right\} \cap Q_{n}}\right] .
\end{aligned}
$$


Again, we observe the symmetry of the distribution of the sizes of the special and nonspecial subtrees, and use the results we developed for $\mathbb{P}\left(\mathcal{R}_{n}\right)$ and $\mathbb{E}\left[X_{n}\right]$. Note that, by conditioning on $U_{n}=1$, the size of the nonspecial subtree is $n-1$ (with $\widetilde{X}_{n-1}$ protected nodes) and the indicator in the last cross-product term survives when the special subtree independently forms a tree with a protected root. That is, $\mathbb{E}\left[\tilde{X}_{n-U_{n}} \mathbf{1}_{\left\{U_{n}=1\right\} \cap Q_{n}}\right]$ simply becomes $\mathbb{E}\left[X_{n-1} \mathbf{1}_{\mathcal{R}_{n-1}}\right]$, and, similarly, when $U_{n}=1, \mathbb{E}\left[\mathbf{1}_{\left\{U_{n}=1\right\} \cap Q_{n}}\right]$ becomes $\mathbb{E}\left[\mathbf{1}_{\mathcal{R}_{n-1}}\right]$. The second moment recurrence takes the form, for $n \geq 3$,

$$
\begin{aligned}
\mathbb{E}\left[X_{n}^{2}\right]= & \frac{2}{n-1} \sum_{k=1}^{n-1} \mathbb{E}\left[X_{k}^{2}\right]+\frac{1}{n-1}+\frac{\Gamma(n-1,-1)}{\mathrm{e} \Gamma(n)}+\frac{2}{n-1} \sum_{k=1}^{n-1} \mathbb{E}\left[X_{k}\right] \mathbb{E}\left[X_{n-k}\right] \\
& +\frac{2}{n-1} \mathbb{E}\left[X_{n-1}\right]-\frac{2}{n-1} \mathbb{E}\left[X_{n-1} \mathbf{1}_{\mathcal{R}_{n-1}}\right] .
\end{aligned}
$$

Let $Y_{n}=X_{n} \mathbf{1}_{\mathcal{R}_{n}}$. We need the expectation of this random variable in the last recurrence. This we can obtain from a recurrence for $\mathbb{E}\left[Y_{n}\right]$ itself. We highlight only the main points in developing and solving this recurrence. For conciseness, we use

$$
c:=\frac{1}{2}-\frac{1}{\mathrm{e}} \text {. }
$$

Lemma 3.1. The expected value of $Y_{n}$ satisfies

$$
\mathbb{E}\left[Y_{n}\right]=\frac{c}{\mathrm{e}} n+O(1) .
$$

Proof. Multiply both sides of (2.1) by $\mathbf{1}_{\mathcal{R}_{n}}$, and take expections to obtain, for $n \geq 3$,

$$
\mathbb{E}\left[Y_{n}\right]=\frac{1}{n-1} \sum_{k=1}^{n-2} \mathbb{E}\left[X_{k}\right] \mathbb{P}\left(\mathcal{R}_{n-k}\right)+\frac{1}{n-1} \mathbb{E}\left[X_{n-1}\right]+\frac{1}{n-1} \sum_{k=1}^{n-2} \mathbb{E}\left[Y_{k}\right]+\frac{1}{n-1}
$$

Using (2.4), we have, for $n \geq 3$,

$$
\mathbb{E}\left[Y_{n}\right]=\frac{1}{n-1} \sum_{k=1}^{n-1} \mathbb{E}\left[X_{k}\right] \frac{\Gamma(n-k,-1)}{\mathrm{e} \Gamma(n-k)}+\frac{1}{n-1} \sum_{k=1}^{n-2} \mathbb{E}\left[Y_{k}\right]+\frac{1}{n-1} .
$$

Differencing yields, for $n \geq 4$,

$$
\begin{aligned}
& (n-1) \mathbb{E}\left[Y_{n}\right]-(n-2) \mathbb{E}\left[Y_{n-1}\right] \\
& \quad=\sum_{k=1}^{n-1} \mathbb{E}\left[X_{k}\right] \frac{\Gamma(n-k,-1)}{\mathrm{e} \Gamma(n-k)}-\sum_{k=1}^{n-2} \mathbb{E}\left[X_{k}\right] \frac{\Gamma(n-1-k,-1)}{\mathrm{e} \Gamma(n-1-k)}+\mathbb{E}\left[Y_{n-2}\right] .
\end{aligned}
$$

We define $b_{n}$, for all $n \geq 0$, as

$$
b_{n}:=\sum_{k=1}^{n-1} \mathbb{E}\left[X_{k}\right] \frac{\Gamma(n-k,-1)}{\mathrm{e} \Gamma(n-k)}-\sum_{k=1}^{n-2} \mathbb{E}\left[X_{k}\right] \frac{\Gamma(n-1-k,-1)}{\mathrm{e} \Gamma(n-1-k)} .
$$


In particular, note that $b_{0}=b_{1}=b_{2}=b_{3}=0$. We have, for $n \geq 4$, the recurrence

$$
(n-1) \mathbb{E}\left[Y_{n}\right]-(n-2) \mathbb{E}\left[Y_{n-1}\right]=b_{n}+\mathbb{E}\left[Y_{n-2}\right] .
$$

This recursive equation can be written in terms of (ordinary) generating functions. We define

$$
B(z):=\sum_{n=0}^{\infty} b_{n} z^{n} \quad \text { and } \quad A(z):=\sum_{n=0}^{\infty} \mathbb{E}\left[Y_{n}\right] z^{n}
$$

where $Y_{0}:=0$ for this purpose. In what follows, we use the coefficient extractor $\left[z^{n}\right] g(z)$, which stands for the $n$th coefficient in the series expansion of a function $g(z)$. So, (3.2) yields, for $n \geq 4$,

$$
\left[z^{n-2}\right]\left(\frac{\mathrm{d}}{\mathrm{d} z}\left(\frac{A(z)}{z}\right)\right)-\left[z^{n-3}\right]\left(\frac{\mathrm{d}}{\mathrm{d} z}\left(\frac{A(z)}{z}\right)\right)=\left[z^{n}\right] B(z)+\left[z^{n-2}\right] A(z),
$$

or, equivalently, for $n \geq 4$,

$$
\left[z^{n}\right]\left(z^{2}\left(\frac{\mathrm{d}}{\mathrm{d} z}\left(\frac{A(z)}{z}\right)\right)-z^{3}\left(\frac{\mathrm{d}}{\mathrm{d} z}\left(\frac{A(z)}{z}\right)\right)-B(z)-z^{2} A(z)\right)=0 .
$$

Moreover, this equation holds for $n$ smaller than 4 too, except at $n=3$. So, we adjust by $z^{3}$, and we obtain a differential equation for these generating functions:

$$
z^{2}\left(\frac{\mathrm{d}}{\mathrm{d} z}\left(\frac{A(z)}{z}\right)\right)-z^{3}\left(\frac{\mathrm{d}}{\mathrm{d} z}\left(\frac{A(z)}{z}\right)\right)-B(z)-z^{2} A(z)-z^{3}=0 .
$$

This is a standard first-order differential equation. It has the solution

$$
A(z)=\frac{\mathrm{e}^{-z} z}{1-z} \int_{0}^{z}\left(\frac{\left(B(t)+t^{3}\right) \mathrm{e}^{t}}{t^{2}}\right) \mathrm{d} t .
$$

Therefore, extracting the coefficient of $z^{n}$, we obtain

$$
\mathbb{E}\left[Y_{n}\right]=\left[z^{n}\right] \frac{\mathrm{e}^{-z} z}{1-z} \int_{0}^{z}\left(\frac{\left(B(t)+t^{3}\right) \mathrm{e}^{t}}{t^{2}}\right) \mathrm{d} t
$$

For $B(t)$, we have

$$
B(t)=\sum_{n=0}^{\infty}\left(\sum_{k=1}^{n-1} \mathbb{E}\left[X_{k}\right] \frac{\Gamma(n-k,-1)}{\mathrm{e} \Gamma(n-k)}-\sum_{k=1}^{n-2} \mathbb{E}\left[X_{k}\right] \frac{\Gamma(n-1-k,-1)}{\mathrm{e} \Gamma(n-1-k)}\right) z^{n},
$$

but $\mathrm{e}^{-1}(\Gamma(a,-1) / \Gamma(a))=\mathrm{e}^{-1}-\sum_{\ell=a}^{\infty}(-1)^{\ell} / \ell !$, so we have

$$
\begin{aligned}
B(t) & =\sum_{n=0}^{\infty}\left(\sum_{k=1}^{n-1} \mathbb{E}\left[X_{k}\right]\left(\mathrm{e}^{-1}-\sum_{\ell=n-k}^{\infty} \frac{(-1)^{\ell}}{\ell !}\right)-\sum_{k=1}^{n-2} \mathbb{E}\left[X_{k}\right]\left(\mathrm{e}^{-1}-\sum_{\ell=n-1-k}^{\infty} \frac{(-1)^{\ell}}{\ell !}\right)\right) t^{n} \\
& =\sum_{n=2}^{\infty} \mathbb{E}\left[X_{n-1}\right] t^{n}+\sum_{n=2}^{\infty}\left(\sum_{k=1}^{n-2} \mathbb{E}\left[X_{k}\right] \frac{(-1)^{n-1-k}}{(n-1-k) !}\right) t^{n} .
\end{aligned}
$$


The closest singularity of $A(z)$ to the origin (treating $z$ as a complex-valued variable) is at $z=1$. Thus, by the methods of analytic combinatorics (see, e.g. Chapter 6 of [4], which discusses singularity analysis of generating functions), we can compute the asymptotic growth of $\mathbb{E}\left[Y_{n}\right]$ by precisely characterizing the behavior of $A(z)$, as $z \rightarrow 1$. To do this, we note that, as $t \rightarrow 1$, we have

$$
\begin{aligned}
B(t)= & \left(\frac{(1 / 2)-\mathrm{e}^{-1}}{(t-1)^{2}}+\frac{1-2 \mathrm{e}^{-1}}{t-1}+O(1)\right) \\
& +\left(\frac{(3 / 2) \mathrm{e}^{-1}-\mathrm{e}^{-2}-(1 / 2)}{(t-1)^{2}}+\frac{-1+(5 / 2) \mathrm{e}^{-1}-\mathrm{e}^{-2}}{t-1}+O(1)\right) \\
= & \frac{(1 / 2) \mathrm{e}^{-1}-\mathrm{e}^{-2}}{(t-1)^{2}}+\frac{(1 / 2) \mathrm{e}^{-1}-\mathrm{e}^{-2}}{(t-1)}+O(1) .
\end{aligned}
$$

It follows immediately that

$$
\begin{aligned}
\mathbb{E}\left[Y_{n}\right]= & {\left[z^{n}\right]\left(\frac{-\mathrm{e}^{-1}}{z-1}+0+O(z-1)\right) } \\
& \times \int_{0}^{z}\left(\frac{(1 / 2) \mathrm{e}^{-1}-\mathrm{e}^{-2}}{(t-1)^{2}}+\frac{(1 / 2) \mathrm{e}^{-1}-\mathrm{e}^{-2}}{(t-1)}+O(1)\right) \\
& \quad \times\left(\mathrm{e}-\mathrm{e}(t-1)+O\left((t-1)^{2}\right)\right) \mathrm{d} t \\
= & {\left[z^{n}\right]\left(\frac{(1 / 2) \mathrm{e}^{-1}-\mathrm{e}^{-2}}{(z-1)^{2}}+O(z-1)\right) } \\
= & \left(\frac{1}{2} \mathrm{e}^{-1}-\mathrm{e}^{-2}\right) n+O(1) .
\end{aligned}
$$

Proposition 3.1. We have

$$
\mathbb{E}\left[X_{n}^{2}\right]=c^{2} n^{2}+O(n)
$$

Proof. By recurrence (3.1), we have, for $n \geq 4$, and differencing,

$$
\begin{aligned}
\mathbb{E}\left[X_{n}^{2}\right]= & \frac{n}{n-1} \mathbb{E}\left[X_{n-1}^{2}\right] \\
& +\frac{(n-1)(\Gamma(n-1,-1)) / \mathrm{e} \Gamma(n)-(n-2)(\Gamma(n-2,-1)) / \mathrm{e} \Gamma(n-1)}{n-1} \\
& +\frac{2}{n-1}\left(\sum_{k=1}^{n-1} \mathbb{E}\left[X_{k}\right] \mathbb{E}\left[X_{n-k}\right]-\sum_{k=1}^{n-2} \mathbb{E}\left[X_{k}\right] \mathbb{E}\left[X_{n-1-k}\right]\right) \\
& +\frac{2}{n-1}\left(\mathbb{E}\left[X_{n-1}\right]-\mathbb{E}\left[X_{n-2}\right]\right)-\frac{2}{n-1}\left(\mathbb{E}\left[Y_{n-1}\right]-\mathbb{E}\left[Y_{n-2}\right]\right) .
\end{aligned}
$$

By the asymptotic representations in Corollary 2.1 and Lemma 3.1, we simplify the latter recurrence to

$$
\begin{aligned}
\mathbb{E}\left[X_{n}^{2}\right] & =\frac{n}{n-1} \mathbb{E}\left[X_{n-1}^{2}\right]+O\left(\frac{1}{n^{2}}\right)+\left(c^{2} n+O\left(\frac{1}{n}\right)\right)+O\left(\frac{1}{n}\right)+O\left(\frac{1}{n}\right) \\
& =\frac{n}{n-1} \mathbb{E}\left[X_{n-1}^{2}\right]+c^{2} n+O\left(\frac{1}{n}\right) .
\end{aligned}
$$

This type of recurrence has the claimed solution. 
Corollary 3.1. We have

$$
\operatorname{var}\left[X_{n}\right]=O(n) .
$$

The relatively small variance allows us to write a concentration law.

Proposition 3.2. We have

$$
\frac{X_{n}}{n} \stackrel{\mathbb{P}}{\rightarrow} c
$$

Proof. Let $\varepsilon>0$ be fixed. By Chebyshev's inequality, we write

$$
\mathbb{P}\left\{\left|\frac{X_{n}}{n}-\frac{\mathbb{E}\left[X_{n}\right]}{n}\right|>\varepsilon\right\} \leq \frac{\operatorname{var}\left[X_{n}\right]}{\varepsilon^{2} n^{2}}=O\left(\frac{1}{n}\right) \rightarrow 0 .
$$

Hence, $(1 / n) X_{n}-(1 / n) \mathbb{E}\left[X_{n}\right] \stackrel{\mathbb{P}}{\rightarrow} 0$. We also know that $(1 / n) \mathbb{E}\left[X_{n}\right]-c \rightarrow 0$. Adding the two convergence relations, the result follows.

\section{Acknowledgements}

This research was carried out while the first author was visiting Purdue University. The support the first author received from Purdue's Department of Statistics is sincerely appreciated. Dr. Mark D. Ward hosted the visit, and his hospitality was way above any expectation. The second author was supported by NSF Science \& Technology Center grant CCF-0939370. The authors wish to express their thanks to Svante Janson for commenting on earlier drafts.

\section{References}

[1] Bergeron, F., Flajolet, P. and Salvy, B. (1992). Varieties of increasing trees. In (CAAP '92) Lecture Notes Comput. Sci. 581, Springer, Berlin, pp. 24-48.

[2] Cheon, G.-S. and Shapiro, L. W. (2008). Protected points in ordered trees. Appl. Math. Lett. 21, 516-520.

[3] Du, R. R. X. And Prodinger, H. (2012). Notes on protected nodes in digital search trees. Appl. Math. Lett. 25, 1025-1028.

[4] Flajolet, P. and Sedgewick, R. (2009). Analytic Combinatorics. Cambridge University Press.

[5] Mahmoud, H. M. and Ward, M. D. (2012). Asymptotic distribution of two-protected nodes in random binary search trees. Appl. Math. Lett. 25, 2218-2222.

[6] Mansour, T. (2011). Protected points in k-ary trees. Appl. Math. Lett. 24, 478-480.

[7] Smythe, R. T. and Mahmoud, H. M. (1995). A survey of recursive trees. Theory Prob. Math. Statist. 51, 1-27.

[8] Van der Hofstad, R., Hooghiemstra, G. and Van Mieghem, P. (2002). On the covariance of the level sizes in random recursive trees. Random Structures Algorithms 20, 519-539. 